\title{
Recursos tecnológicos emergentes como herramientas didácticas para el área de Matemáticas en Educación Básica Superior
}

\section{Emerging technological resources as didactic tools for the area of Mathematics in Higher Basic Education}

\author{
Rosa Maricela Palaguachi-Álvarez \\ rosa.palaguachi.68@est.ucacue.edu.ec \\ Universidad Católica de Cuenca, Biblián \\ Ecuador \\ https://orcid.org/0000-0002-0096-7479 \\ Darwin Gabriel García-Herrera \\ dggarciah@ucacue.edu.ec \\ Universidad Católica de Cuenca, Azogues \\ Ecuador \\ https://orcid.org/0000-0001-6813-8100 \\ Sandra Elizabeth Mena-Clerque \\ sandramena@ucacue.edu.ec \\ Universidad Católica de Cuenca, Cuenca \\ Ecuador \\ https://orcid.org/0000-0002-9186-2161 \\ Juan Carlos Erazo-Álvarez \\ jcerazo@ucacue.edu.ec \\ Universidad Católica de Cuenca, Cuenca \\ Ecuador \\ https://orcid.org/0000-0001-6480-2270
}

Recepción: 10 agosto 2020 Revisado: 25 septiembre 2020

Aprobación: 15 octubre 2020

Publicación: 01 noviembre 2020 


\title{
RESUMEN
}

El objetivo fue analizar los recursos tecnológicos emergentes como herramientas didácticas para el área de Matemáticas en Educación Básica Superior. se desarrolló desde un diseño no experimental y tipo descriptivo, se contó con la participacion de estudiantes y docentes de la básica superior de diferentes instituciones educativas de la provincia del Azuay - Ecuador, por lo cual se empleó un muestreo por conglomerados, constituyéndose una muestra de 68 estudiantes y 23 docentes. el $73,53 \%$ de los estudiantes manifestaron que el uso de recursos tecnológicos facilita su proceso de enseñanza-aprendizaje y a su vez instaron a que los docentes implementen en su quehacer educativo diversos recursos que les permita adentrasen aun espacio más real y significativo a fin de consolidar y afianzar sus conocimientos. Se plantearon herramientas digitales como CalcMec, Desmos y Symbaloo, recursos que requieren que los actores estén a la vanguardia en esta nueva sociedad online.

Descriptores: Matemáticas; tecnología de la información; informática educativa. (Palabras tomadas del Tesauro UNESCO).

\begin{abstract}
The objective was to analyze emerging technological resources as didactic tools for the area of Mathematics in Higher Basic Education. It was developed from a non-experimental design and descriptive type, with the participation of students and teachers of the upper basic of different educational institutions of the province of Azuay - Ecuador, for which a cluster sampling was used, constituting a sample of 68 students and 23 teachers. $73.53 \%$ of the students stated that the use of technological resources facilitates their teachinglearning process and in turn urged that teachers implement in their educational work various resources that allow them to enter even more real and meaningful space in order to consolidate and strengthen their knowledge. Digital tools such as CalcMec, Desmos and Symbaloo were proposed, resources that require actors to be at the forefront in this new online society.
\end{abstract}

Descriptors: Mathematics; information technology; computer uses in education. (Words taken from the UNESCO Thesaurus). 


\section{INTRODUCCIÓN}

Uno de los requisitos fundamentales en la formación de los estudiantes de educación básica ha sido y será el área de matemáticas, sin embargo el aprendizaje de esta área desde años anteriores se ha convertido en un verdadero problema, ya que según el Programa para la Evaluación Internacional de Alumnos (PISA, por sus siglas en inglés) de la Organización para la Cooperación y Desarrollo Económico (OCDE) realizada en el año 2018 dio como resultado que en América Latina los estudiantes escasamente alcanzaron un promedio de 489, y en lo que respecta al Ecuador él $69 \%$ de los estudiantes no alcanzaron el nivel básico de habilidades. Así mismo en las pruebas ser bachiller evaluados por el Instituto Nacional de Evaluación Educativa (INEVAL) en lo que se refiere al año lectivo 2019-2020, los estudiantes en la región Sierra Interandina difícilmente alcanzaron una nota promedio de 743 considerado este, como un nivel de logro elemental.

De acuerdo a estos datos el área de matemáticas está considerada como una de las áreas más bajas en promedios de competencia. Lo cual conlleva a la necesidad de optimizar, dinamizar y facilitar el proceso de enseñanza-aprendizaje, mediante la utilización de recursos tecnológicos didácticos e interactivos que despierten el interés y la motivación de los estudiantes por el área. En tal sentido se procura la vinculación de las Tecnologías de la Información y Comunicación (TIC) en la enseñanza, puesto que estos medios contribuyen al desarrollo de escenarios más realistas y prácticos, donde los estudiantes pueden desarrollar habilidades de exploración, representación e interpretación.

Por lo tanto, resulta fundamental la utilización de recursos tecnológicos e innovadores en el ámbito escolar sobre todo en aquellas áreas en las cuales existe una problemática tan marcada como es el caso de matemáticas (Alvites, 2017). De ahí que en los últimos años también se viene buscando un cambio en el rol del docente, en el cual se demanda que se convierta en el mediador del aprendizaje, que utilice nuevas estrategias metodológicas 
a favor de una educación más activa, que pueda dar respuesta a los cambios cada vez más profundos y vertiginosos del mundo global de hoy (Torres \& Cobo, 2017).

Por su parte el Gobierno Nacional del Ecuador, en su afán de vincular las TIC en las aulas de clase, dictaminó en el artículo 6, inciso j, de la Ley Orgánica de Educación Intercultural (LOEI), garantizar la alfabetización digital y el uso de las tecnologías de la información y comunicación en los procesos de enseñanza-aprendizaje, con el objetivo de incorporar a la comunidad educativa a la sociedad del conocimiento (MINEDUC, 2015).

Por todo esto, la presente investigación tiene como objetivo fundamental fomentar en los docentes la utilización de recursos tecnológicos didácticos e innovadores para optimizar y enriquecer el proceso de enseñanza- aprendizaje del área de matemática, en básica superior, mediante el empleo de prácticas innovadoras mediadas por las TIC, actualmente resulta fundamental dotar a los docentes de nuevas estrategias y competencias tecnológicas, que les permita dejar atrás la enseñanza tradicional, se innoven y estén acorde a las exigencias del mundo actual del hoy (Domínguez, et al., 2017).

\section{Referencial teórico}

Actualmente los recursos tecnológicos se han convertido en una herramienta importante en la educación, por cuanto son una necesidad diaria para el proceso de enseñanza aprendizaje, estimulan el proceso del inter-aprendizaje, permitiéndole al estudiante estar lo más cercano posible a la realidad y en el caso de los docentes permite transformar el salón de clases en un potente escenario virtual para procesos pedagógicos. De esta manera la utilización de tabletas, laptops, celulares Android, la tele servicios ha permitido tener un acercamiento sincrónico y asincrónico entre docentes y estudiantes, lo cual permite y facilita transportarse a espacios de aprendizaje desde cualquier sitio y en cualquier momento, estimulando la imaginación y el interés por el aprendizaje. 
Otros recursos tecnológicos usados en la educación, es el uso de material audiovisual sirviendo como complemento a los medios de comunicación clásicos de la enseñanza estos son: proyectores, vídeos, películas, audios, softwares, programas informáticos (DVD, Pendrive), multimedia e internet, páginas web, weblogs, webquest, correo electrónico, chats, foros entre otros.

De ahí que el empleo de los recursos tecnológicos interactivos permite articular los contenidos de las clases teóricas, con clases experimentales, facilitando así el proceso de enseñanza-aprendizaje. Por ello desde hace cuarenta años el uso de las TIC en la educación ha tenido una importante y significativa evolución, afianzada en la teoría conductista, cognitiva y la reciente teoría sociocultural (López-Neira, 2017). Cada una de estas teorías han permitido ver las transformaciones que se han dado en el contexto educativo a partir de la incorporación de las tecnologías digitales y el uso del computador. Sin embargo, la matemática ha sido uno de los campos del saber que más se ha rezagado, ya sea por concepciones tradicionalistas y poco objetivas o por un profundo rechazo al área debido a los problemas que suelen presentar los educandos al momento de su estudio.

No obstante, es indiscutible que el estudio de la matemática no es un proceso simple y en los distintos contextos de formación se requiere actualizar los métodos de enseñanza e incorporar nuevas estrategias, que permitan a los estudiantes adquirir hábitos de estudio, reconocer los alcances que tiene la matemática en una vida profesional y práctica (Soler-Contreras, et al., 2017). De ahí que el uso de currículos estructurados y secuenciales han servido para adquirir habilidades procedimentales y fundamentales, el proceso de aprendizaje de esta área va más allá, lo cual demanda la integración de recursos tecnológicos innovadores como soporte a la enseñanza-aprendizaje (TrianaMuñoz, et al., 2016). 
María-del-Carmen García-Cárdenas; Darwin Gabriel García-Herrera; Nancy Marcela Cárdenas-Cordero; Juan Carlos Erazo-Álvarez

Un estudio realizado en México a 120 estudiantes, en el que se utilizó recursos tecnológicos como sistema de aprendizaje en línea para la enseñanza de matemáticas en educación básica, el e-Collaborative Learning Sistema Integral Colaborativo para la educación sin Barreras (SICEB) ), demostró que el desempeño realizado a través de esta plataforma virtual promovió la formación integral de los estudiantes, mejoró la capacidad de estructurar ideas, incrementó el análisis, el trabajo colaborativo y la resolución de problemas. Por lo que se reconoció como una herramienta ideal para potenciar el aprendizaje (Barrera-del-Castillo, et al., 2015).

Otro estudio realizado en Buenos Aires en el cual se implementó GeoGebra como recurso tecnológico para la enseñanza de gráficas y funciones en la educación secundaria, manifestó que el uso regular de GeoGebra permitió desarrollar en los estudiantes conocimientos matemáticos e informáticos, además de contextualizar diversos objetos geométricos, resultando de esta manera una herramienta no solo de visualización, sino también de representación (Novembre, et al., 2015).

Por su parte (González-Ogando, 2020), enfatiza la utilización de Desmos, herramienta tecnológica en línea, utilizada para la representación de gráficas y funciones, en la educación secundaria, manifestando que la herramienta contribuye a mejorar la construcción e interpretación de funciones y también resulta de ayuda al profesorado ya que esta es capaz de reducir las dificultades que muchos estudiantes presentan al extraer la información por lo cual alienta a la docencia a utilizar este tipo de herramienta.

\section{Recursos tecnológicos innovadores}

La tecnología es un medio que ofrece grandes recursos en el proceso educativo como estrategias para la innovación dentro del proceso de enseñanza-aprendizaje, sin embargo, el reto es aprender a usarlos y para ello hay que tomar la decisión de utilizarlos, puesto que, es el docente quien debe replantear sus clases y orientarlas hacia el uso de recursos tecnológicos innovadores. (Jiménez-García \& Jiménez-Izquierdo, 2017) 
sostienen que innovar la educación significa introducir en sus técnicas de enseñanza la utilización de la tecnología. Sin embargo, el aprendizaje de matemáticas es un verdadero desafío, ya que esta área, no solo es compleja para aprender sino también para enseñar. Actualmente existen diversas herramientas que se encuentran en la web y que fácilmente pueden convertirse en recursos virtuales de aprendizaje, es evidente que los recursos tecnológicos están creando una gran transición para la nueva forma de enseñar, especialmente en el nivel de la básica superior en la cual es posible utilizar recursos tecnológicos que los estudiantes poseen y convertirlos en un instrumento de aprendizaje, a través del cual los estudiantes pueden aprender desde otras perspectivas, de forma sincrónica y asincrónica facilitando y dinamizando el aprendizaje (Grisales-Aguirre, 2018). Sin embargo, esto debe darse de manera articulada entre lo técnico y lo pedagógico, pues no se puede caer en un simple uso instrumental y dejar de lado la didáctica y la construcción de conocimientos mediada por el docente instructor.

\section{Herramientas didácticas para el área de matemática en básica superior.}

Sabiendo que matemática es una asignatura abstracta y aplicada, hoy en día la gran cantidad de programas online, plataformas educativas, software libre, las redes sociales y el Internet han permitido que las instituciones educativas aprovechen estas herramientas digitales como estrategias para la enseñanza, sin embargo, la falta de capacitación de los docentes dentro de las competencias tecnológicas hace que muy pocos docentes implementen dichas herramientas en su quehacer educativo.

Por ello es importante, establecer un reto de cambio de paradigma ya que el progreso de los recursos tecnológicos está tomando gran auge en distintos sectores, no solo en el ámbito académico, sino también en muchos e importantes sectores de la sociedad. En este sentido, el cambio pedagógico solo será posible si se cruza la perspectiva de innovación tecnológica, en concordancia con el diseño y utilización de auténticas 
herramientas didácticas que permitan crear entornos virtuales de aprendizaje a fin de mejorar la calidad de la enseñanza (Delgado-García, et al., 2018).

Por consiguiente, la labor del docente consiste en elaborar materiales didácticos y tecnológicos de tal manera que sean relevantes y significativos para sus educandos, es decir convertirse en un auténtico diseñador de medios, lo cual implica que realice diferentes tareas desde el punto de vista instrumental (manejo de herramientas y recursos) y operacional (conexión al manejo de páginas web, blog, entre otros). En suma, todas estas técnicas, habilidades, que el docente debe conocer se enmarcan en to pedagógico, en donde el docente es el planificador, organizador, guía, conciliador, evaluador, moderador, del proceso. Además de ello, debe ser inclusivo, capaz de crear entornos de aprendizaje cooperativos y colaborativos. Por lo que, el docente además de tener conocimientos competentes y habilidades en el empleo de las tecnologías; debe crear espacios armónicos, donde los estudiantes puedan sentirse a gusto y utilizar la tecnología como medio de aprendizaje.

A su vez, la matemática al ser una ciencia que está representada con números, símbolos, figuras geométricas, graficas cartesianas demanda el uso de diversos recursos tecnológicos. (Arévalo-Duarte \& Gamboa-Suárez, 2015) refieren que a nivel mundial existe la necesidad de establecer lineamientos claros y concretos para el uso de estos recursos tecnológicos con el fin de crear una sociedad más interconectada a las nuevas tecnologías. En este sentido el Ministerio de Educación del Ecuador, desde hace años viene promulgando la integración de recursos tecnológicos en los procesos de aprendizaje, en correspondencia con los contenidos establecidos en el currículo, con el fin de mejorar e innovar la calidad educativa.

De esta manera, de acuerdo a lo establecido en la malla curricular del sistema educativo ecuatoriano, se fomenta la utilización de las siguientes herramientas tecnológicas en la básica superior, mismas que tienen vinculación directa con los contenidos de aprendizaje de matemáticas. 
María-del-Carmen García-Cárdenas; Darwin Gabriel García-Herrera; Nancy Marcela Cárdenas-Cordero; Juan Carlos Erazo-Álvarez

\section{Tabla 1.}

Herramientas didácticas para el área de matemáticas, básica superior.

\begin{tabular}{lll}
\hline \multicolumn{1}{c}{ Categoría } & Aplicación & \multicolumn{1}{c}{ Descripción } \\
\hline Funciones y gráficas: & Desmos & $\begin{array}{l}\text { Aplicación online para representar y estudiar } \\
\text { funciones de forma gráfica. Cuenta con una } \\
\text { base de datos de actividades. }\end{array}$ \\
Álgebra & CalcMe & $\begin{array}{l}\text { Aplicación online que permite resolver y } \\
\text { construir todo tipo de expresiones } \\
\text { algebraicas. }\end{array}$ \\
Aprendizaje en línea & Symbaloo & $\begin{array}{l}\text { Plataforma gratuita basada en la nube, } \\
\text { permite a los usuarios organizar y categorizar } \\
\text { planes de clase, de forma personalizada y } \\
\text { organizada mediante las cuales los docentes } \\
\text { pueden enviar. }\end{array}$
\end{tabular}

Fuente: Coloma, et al. (2020).

Cabe enfatizar que estas herramientas digitales se fundamentan en el constructivismo, en donde los aprendices exploran y desarrollan su propio aprendizaje, sin embargo, son los docentes los responsables de la integración y uso de estos recursos, estos modelos didácticos están destinados para innovar y luego enseñar, con base en el aprendizaje colaborativo, participativo y autónomo.

\section{METODOLOGÍA}

La presente investigación se desarrolló desde un diseño no experimental y tipo descriptivo, a partir de un enfoque cuantitativo, de cohorte transversal, por cuanto los datos fueron recabados en un solo periodo de tiempo, se contó con la participacion de estudiantes y docentes de la básica superior de diferentes instituciones educativas de la 
provincia del Azuay - Ecuador, por lo cual se empleó un muestreo por conglomerados, constituyéndose una muestra de 68 estudiantes y 23 docentes.

Se aplicó una encuesta estructurada online tanto a los estudiantes como a los docentes, fueron digitalizadas en la plataforma Google Forms y constaron de 8 ítems cada una, se empleó el Software SPSS para validar los datos de las encuestas, mismas que realizado el análisis de fiabilidad de Alfa de Cronbach fue de 0.714 en las encuestas de los estudiantes, mientras que en las encuestas de los docentes fue de 0.726 de fiabilidad en el Alfa de Cronbach.

A raiz de las encuestas, se recolectó datos estadísticos significativos, referentes al fenómeno de la investigación, de tal manera que fue posible conocer y analizar como actuan los docentes y estudiantes en sus aulas de clase. De ahí, que, de acuerdo al número de estudiantes encuestados, que fue de 68, se usó el test Kolmogorov para diferenciar la normalidad del conjunto de datos, en donde se obtuvo un valor de 0,00 con lo cual se logró determinar que todos los datos fueron paramétricos. De igual manera como el número de docentes encuestados fue de 23 se usó el test Shapiro Wilk para constatar la normalidad del conjunto de datos y así mismo se obtuvo un valor de 0,00 en todos los datos, con lo cual se alcanzó a establecer que todos los datos fueron paramétricos y por ende se asumió que todas las variables fueron normales ( $\mathrm{Ho})$.

\section{RESULTADOS}

Una vez recolectados y procesados los datos de los docentes, se realizó en primer lugar un análisis estadístico descriptivo de frecuencias, en el cual se pudo constatar que un $73,91 \%$ docentes encuestados no estaban capacitados para trabajar de forma virtual. 
María-del-Carmen García-Cárdenas; Darwin Gabriel García-Herrera; Nancy Marcela Cárdenas-Cordero; Juan Carlos Erazo-Álvarez

\section{Tabla 2.}

Docentes estaban capacitados para trabajar de forma virtual.

\begin{tabular}{cccccc}
\hline & Frecuencia & Porcentaje & $\begin{array}{c}\text { Porcentaje } \\
\text { válido }\end{array}$ & $\begin{array}{c}\text { Porcentaje } \\
\text { acumulado }\end{array}$ \\
\hline \multirow{4}{*}{ Válidos } & Muy en desacuerdo & 1 & 4.3 & 4.3 & 4.3 \\
& En desacuerdo & 17 & 73.9 & 73.9 & 78.3 \\
& De acuerdo & 4 & 17.4 & 17.4 & 95.7 \\
\cline { 2 - 6 } & Totalmente de acuerdo & 1 & 4.3 & 4.3 & 100.0 \\
\cline { 2 - 6 } & Total & 23 & 100.0 & 100.0 & \\
\cline { 2 - 6 }
\end{tabular}

De igual manera, bajo los mismos parámetros se logró evidenciar que todos los docentes encuestados requieren, más conocimientos sobre herramientas tecnológicas innovadoras para impartir el área de matemáticas, puesto que el 56,52\% está de acuerdo y el $43,46 \%$ está totalmente de acuerdo. Con lo cual el $100 \%$ de docentes encuestados manifestaron que demandan de más competencias tecnológicas en su quehacer educativo.

Así mismo, se realizó un análisis de relación entre variables con Chi-cuadrado, en la cual se plantearon las siguientes hipótesis:

Ho: No existe relación entre el uso de recursos tecnológicos y la necesidad de requerir más conocimientos sobre herramientas tecnológicas innovadoras para impartir el área de matemáticas.

H1: Existe relación entre el uso de recursos tecnológicos y la necesidad de requerir más conocimientos sobre herramientas tecnológicas innovadoras para impartir el área de matemáticas. 
María-del-Carmen García-Cárdenas; Darwin Gabriel García-Herrera; Nancy Marcela Cárdenas-Cordero; Juan Carlos Erazo-Álvarez

\section{Tabla 3.}

Relación entre el uso de recursos tecnológicos y la necesidad de requerir más conocimientos sobre herramientas tecnológicas innovadoras para el área de matemáticas.

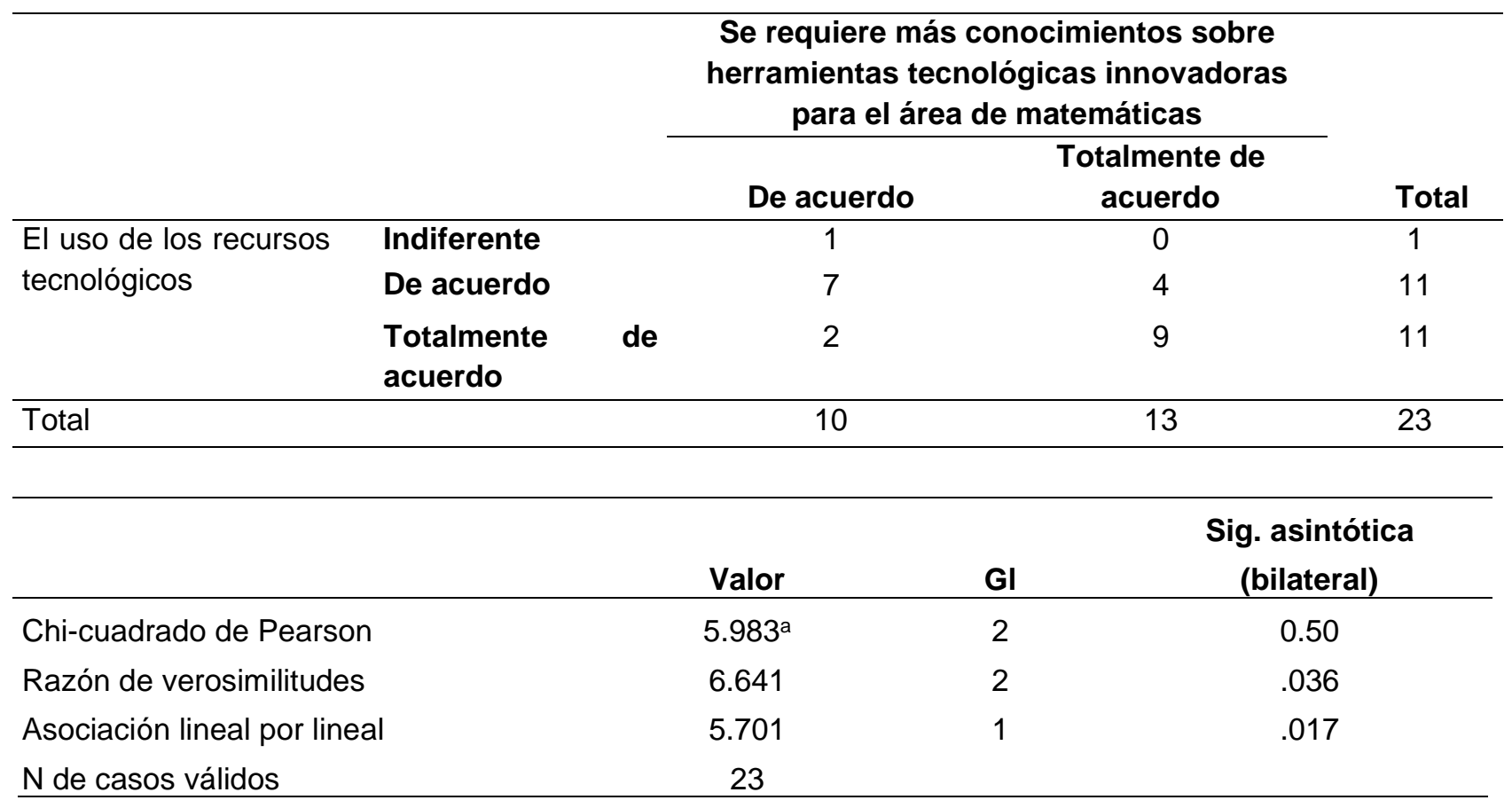

a. 4 casillas $(66.7 \%)$ tienen una frecuencia esperada inferior a 5. La frecuencia mínima esperada es .43.

Fuente: Elaboración propia.

En la tabla 3, se alcanzó evidenciar que el valor de la Sig. Bilateral para el Chi-cuadrado de Pearson es 0,05 por lo que se asume a la hipótesis como afirmativa, misma que a su vez, determina que existe relación entre la utilización de recursos tecnológicos y la necesidad de requerir más conocimientos sobre herramientas tecnológicas innovadoras para impartir el área de matemáticas. Con lo cual se determinó que los docentes necesitan conocer más recursos tecnológicos innovadores para implementar en sus procesos de aprendizaje.

El $73,53 \%$ de estudiantes encuestados están totalmente de acuerdo que el uso de recursos tecnológicos facilita su proceso de aprendizaje de matemáticas y un 22, $10 \%$ 
están de acuerdo. Lo cual consolida que el uso de recursos tecnológicos en las aulas de aprendizaje facilita el cálculo y esquematización de funciones matemáticas.

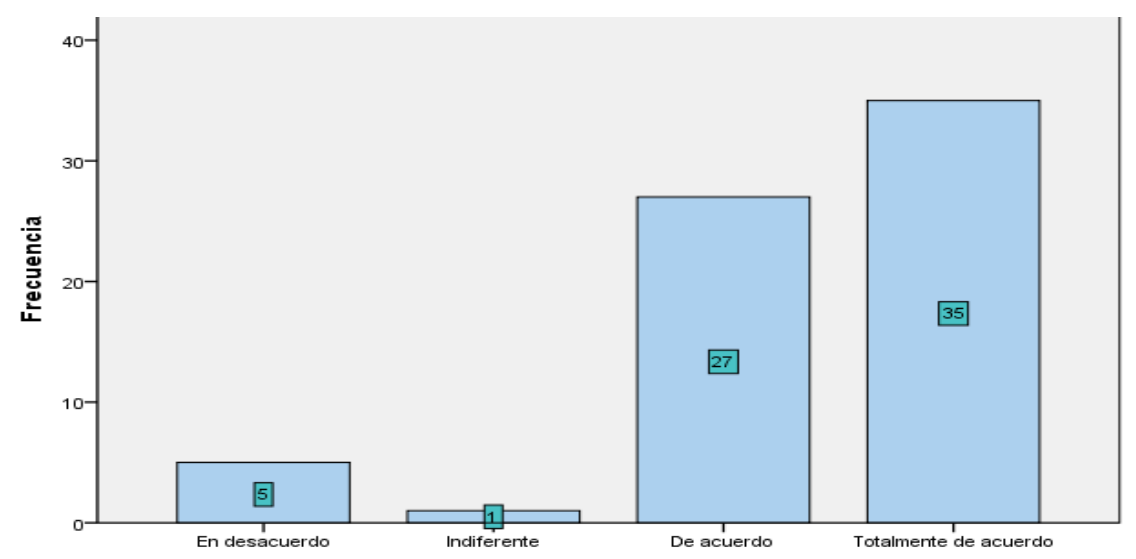

Figura1. Estudiantes que están de acuerdo que su docente implemente herramientas tecnológicas en el proceso de aprendizaje de matemáticas.

De igual manera, bajo los mismos parámetros, en la figura 1, se consiguió evidenciar que 35 estudiantes están totalmente de acuerdo que su docente implemente herramientas digitales innovadoras en el proceso de aprendizaje y así mismo que 27 de ellos, están de acuerdo, dando un total de 62 estudiantes de 68 en total, que manifiestan que requieren que su docente incorpore herramientas tecnológicas innovadoras en su proceso de formación disciplinar. Ante lo cual se vuelve inminente la necesidad de que, el docente conozca y aplique nuevas metodologías tecnológicas. Por otro lado, se realizó un análisis de relación entre variables con Chi-cuadrado, planteándose las siguientes hipótesis:

Ho: No existe relación entre los estudiantes de básica superior y el considerar que el docente debe ser creativo y utilizar diferentes recursos tecnológicos para plantear sus contenidos.

H1: Existe relación entre los estudiantes de básica superior y el considerar que el docente debe ser creativo y utilizar diferentes recursos tecnológicos para plantear sus contenidos. 
María-del-Carmen García-Cárdenas; Darwin Gabriel García-Herrera; Nancy Marcela Cárdenas-Cordero; Juan Carlos Erazo-Álvarez

\section{Tabla 4.}

Relación entre los estudiantes de básica superior y el considerar que el docente debe ser creativo y utilizar diferentes recursos tecnológicos para desarrollar sus contenidos.

\begin{tabular}{|c|c|c|c|c|c|c|}
\hline & & \multicolumn{4}{|c|}{$\begin{array}{l}\text { Consideras el docente debe ser creativo y utilizar } \\
\text { diferentes recursos tecnológicos para desarrollar sus } \\
\text { temas. }\end{array}$} & \multirow[b]{2}{*}{ Total } \\
\hline & & $\begin{array}{c}\text { Muy en } \\
\text { desacuerdo }\end{array}$ & $\begin{array}{c}\text { En } \\
\text { desacuerdo }\end{array}$ & $\begin{array}{c}\text { De } \\
\text { acuerdo }\end{array}$ & $\begin{array}{l}\text { Totalmente } \\
\text { de acuerdo }\end{array}$ & \\
\hline & 8vo. Año & 0 & 0 & 10 & 5 & 15 \\
\hline \multirow[t]{2}{*}{ Año de básica } & 9no. Año & 0 & 3 & 12 & 17 & 32 \\
\hline & 10mo. Año & 1 & 0 & 4 & 16 & 21 \\
\hline \multirow[t]{2}{*}{ Total } & & 1 & 3 & 26 & 38 & 68 \\
\hline & & & alor & GI & \multicolumn{2}{|c|}{$\begin{array}{c}\text { Sig. asintótica } \\
\text { (bilateral) }\end{array}$} \\
\hline \multicolumn{2}{|c|}{ Chi-cuadrado de Pearson } & \multicolumn{2}{|c|}{$13.769^{a}$} & 6 & \multicolumn{2}{|c|}{0.32} \\
\hline \multicolumn{2}{|c|}{ Razón de verosimilitudes } & \multicolumn{2}{|c|}{14.983} & 6 & \multicolumn{2}{|c|}{.020} \\
\hline \multicolumn{2}{|c|}{ Asociación lineal por lineal } & \multicolumn{2}{|c|}{1.199} & 1 & \multicolumn{2}{|c|}{.273} \\
\hline \multicolumn{2}{|c|}{$\mathrm{N}$ de casos válidos } & \multicolumn{2}{|c|}{68} & & & \\
\hline
\end{tabular}

a. 6 casillas $(50.0 \%)$ tienen una frecuencia esperada inferior a 5. La frecuencia mínima esperada es .22.

En la tabla 4, se logró evidenciar que el valor de la Sig. bilateral para el Chi-cuadrado de Pearson es menor que 0,05 , por lo que se asume que la hipótesis es afirmativa, es decir que existe relación entre los estudiantes de básica superior y el considerar que su docente debe ser creativo y utilizar diferentes recursos tecnológicos para desarrollar sus contenidos. De ahí que los estudiantes de básica superior manifiestan que su docente debe estar acorde a las nuevas tendencias tecnológicas y crear entornos virtuales óptimos para el aprendizaje 
María-del-Carmen García-Cárdenas; Darwin Gabriel García-Herrera; Nancy Marcela Cárdenas-Cordero; Juan Carlos Erazo-Álvarez

\section{Tabla 5.}

Relación entre los recursos utilizados por el docente en aula de clases y la importancia de herramientas digitales innovadoras para el proceso de aprendizaje de matemáticas.

\begin{tabular}{|c|c|c|c|c|c|c|}
\hline & & \multicolumn{4}{|c|}{$\begin{array}{c}\text { Importancia de herramientas digitales innovadoras en } \\
\text { el proceso de aprendizaje de matemáticas. }\end{array}$} & \multirow[b]{2}{*}{ Total } \\
\hline & & En desacuerdo & Indiferente & $\begin{array}{c}\text { De } \\
\text { acuerdo }\end{array}$ & $\begin{array}{l}\text { Totalmente } \\
\text { de acuerdo }\end{array}$ & \\
\hline \multirow{3}{*}{$\begin{array}{l}\text { Recursos y utilizados } \\
\text { por el docente en el } \\
\text { aula de clases }\end{array}$} & En desacuerdo & 3 & 0 & 0 & 0 & 3 \\
\hline & De acuerdo & 1 & 1 & 8 & 5 & 15 \\
\hline & $\begin{array}{l}\text { Totalmente } \\
\text { acuerdo }\end{array}$ & 1 & 0 & 19 & 30 & 50 \\
\hline Total & & 5 & 1 & 27 & 35 & 68 \\
\hline
\end{tabular}

\begin{tabular}{lccc}
\hline & Valor & GI & $\begin{array}{c}\text { Sig. asintótica } \\
\text { (bilateral) }\end{array}$ \\
\hline Chi-cuadrado de Pearson & $45.651^{\mathrm{a}}$ & 6 & 0.00 \\
Razón de verosimilitudes & 23.791 & 6 & .001 \\
Asociación lineal por lineal & 25.175 & 1 & .000 \\
N de casos válidos & 68 & & \\
\hline
\end{tabular}

a. 8 casillas $(66.7 \%)$ tienen una frecuencia esperada inferior a 5. La frecuencia mínima esperada es .04.

Fuente: Elaboración propia.

En la tabla 5, se puede observar un encuentro de variables, en la cual se alcanzó a obtener un valor de 0.00 de la Sig. bilateral para el Chi-cuadrado de Pearson, mismo que siendo menor que 0,05 , se admite a la hipótesis como afirmativa, es decir existe relación entre los recursos utilizados por el docente en aula de clases y la importancia de herramientas digitales innovadoras para el proceso de aprendizaje de matemáticas.

Todos estos datos fortalecen la hipótesis inicial al considerar que el uso de recursos tecnológicos innovadores son fundamentales en el proceso de aprendizaje de matemáticas como herramientas didácticas, que permitan fomentar el aprendizaje y a su 
vez despertar el interés y la motivación del estudiantado, estos recursos tecnológicos además de proporcionar nuevos estilos de aprendizaje permiten tener una relación sincrónica y asincrónica entre el docente y el estudiante, mecanismos incuestionables en esta nueva era de educación online.

En base a este aspecto, una vez terminado el análisis estadístico de la investigación, se comparó los resultados obtenidos con otras investigaciones realizadas en diferentes contextos. Tal es el caso de (Marcos, 2016) quien, en su investigación de fin de Master de la Universidad Internacional de la Rioja de Madrid, planteó la utilización de un recurso tecnológico en línea como es la calculadora grafica Desmos, para la enseñanza especifica de funciones y gráficas en educación secundaria. El autor en cuestión manifestó que los resultados obtenidos en su estudio fueron imponderables debido al alto grado de significatividad que tuvo por su carácter visual e interactivo en donde los estudiantes se fueron familiarizando con los contenidos mediante la construcción interactiva de gráficas, permitiendo además al docente jugar con los distintos conceptos y ganar tiempo en la fase expositiva de los temas.

Por otra parte, un estudio realizado en Ecuador concretamente en la ciudad de Loja por (Coloma, et al., 2020) en el cual propusieron la utilización de diferentes recursos en línea como CalcMe para facilitar el aprendizaje de matemáticas en educación básica, dedujeron que la utilización de este recurso tuvo una gran aceptabilidad, por cuanto a los docentes les resulto atractivo y eficiente dicha herramienta debido a sus diversos ítems didácticos y applets de cálculo matemático, con el cual el estudiantado logro desarrollar de forma factible diversas habilidades matemáticas.

Así mismo otra investigación realizada en Ecuador, en la cual se implementó como recurso tecnológico GeoGebra para el área de matemáticas en básica superior, dejo entre ver, que tuvo gran impacto significativo, ya que el promedio del curso subió dos puntos, en relación con la metodología anterior, además de que en el desarrollo del proyecto fue notoria la motivación de los estudiantes, pues se sintieron apoyados en el 
recurso y lograron trazar gráficas y funciones con mediciones exactas de forma clara, dinámica y precisa (Mora, 2020).

De acuerdo, el criterio de los autores vivimos en el mundo de las "Tecnologías de la Información y Comunicación" (TIC), no obstante, la integración de los recursos tecnológicos en la educación sigue siendo un desafío debido a la falta de preparación y formación en conocimientos que debe tener el docente. En tal razón, se exterioriza la siguiente propuesta, la cual pretende fortalecer las oportunidades de aprendizaje de los docentes mediante la descripción de recursos tecnológicos didácticos e innovadores para el área de matemáticas en básica superior.

\section{PROPUESTA}

Tomando como referencia los datos obtenidos en el análisis estadístico de la investigación, se plantea la siguiente propuesta, misma que pretende vincular los contenidos conceptuales del currículo nacional vigente de matemáticas con el uso de recursos tecnológicos didácticos e innovadores para básica superior, pues se ambiciona que estos medios ayuden a mejorar el rendimiento académico y la motivación del estudiantado por el área, ya que actualmente existe un desinterés y falta de atención, que ha provocado que los estudiantes tengan competencias académicas bajas lo cual se ha visto reflejado en las pruebas PISA a nivel internacional y las pruebas ser bachiller a nivel nacional. A continuación, se presenta un gráfico que expone de forma sintética, cada uno de los recursos y sus fases: 


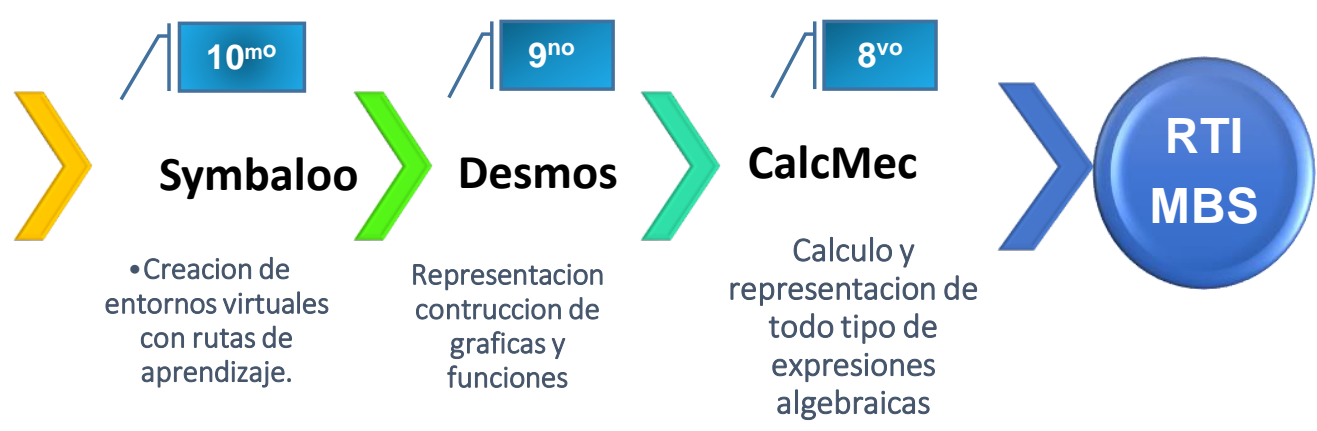

Figura 2. Esquema de recursos tecnológicos innovadores para el de matemáticas, básica superior.

Fuente: Elaboración propia.

CalcMec: Es una calculadora en línea, totalmente gratuita, que funciona desde cualquier navegador y sistema operativo, incluyendo dispositivos móviles y tabletas. Posee un potente sistema de cálculo y dibujo, por lo cual se vuelve un excelente recurso para la enseñanza de matemáticas en la básica superior, concretamente el octavo año de básica, en el cual, según lo establecido en el currículo nacional vigente del MINEDUC, se debe formar al estudiante para la resolución de operaciones y problemas con el conjunto de números reales, expresiones algebraicas, ecuaciones e inecuaciones, descomposición factorial entre otros. Contenidos que generalmente suelen ser de difícil compresión y generar inconvenientes en el estudiante. Frente a esto, es necesario el empleo de la calculadora CalcMec, un recurso alternativo que facilita y dinamiza la comprensión del tema, se encuentra en la siguiente dirección https://calcme.com/a, su utilización es notablemente sencilla y hasta intuitiva, ya que cuenta con demos interactivas, en los cuales se pueden encontrar diversas operaciones, tablas de referencia, ideales para la enseñanza y consolidación de cada tema en particular. 
Desmos: De igual manera Desmos, es una calculadora gráfica online, que está accesible desde cualquier tipo de dispositivo u ordenador, no requiere instalación y posee diferentes idiomas por lo cual puede ser utilizada en distintos contextos. Dando continuidad a lo establecido en el currículo, en el noveno y décimo año de la básica superior, se prevé la enseñanza de los diferentes tipos de funciones para los cuales se pretende que el docente haga uso de este recurso que permitirá que sus estudiantes experimenten por ellos mismos los contenidos desarrollados, fomentando su creatividad y la adquisición de aprendizajes significativos.

La utilización de la herramienta es sencilla y espontánea, basta con ir a la siguiente dirección https://www.desmos.com/ y al momento se presentara la pantalla inicial de Desmos, en la cual se encontrara el plano cartesiano y dando clic sobre el icono del teclado, este se activara y permitirá construir la función o grafica que se requiera. Así mismo cuenta con una gama de demos interactivos en donde se podrá visualizar ejemplos predefinidos que permitirán reforzar los conocimientos tratados en clases.

Symbaloo: Finalmente con Symbaloo, que también es una herramienta online, totalmente gratuita y que puede ser utilizado desde cualquier dispositivo $u$ ordenador, se orienta a crear y personalizar entornos virtuales de aprendizaje, específicamente para los estudiantes del décimo año de la básica superior, puesto que los mencionados considerados nativos digitales se encuentran en la edad ideal, en la cual pueden acceder al recurso y a su vez convertirlo en un espacio virtual de aprendizaje sincrónico asincrónico.

En tal razón se plantea a los docentes a utilizar este recurso, que es dinámico, didáctico y de fácil empleo, se encuentra en la siguiente dirección https://symbalooedu.es/ y a para utilizarlo se debe crear una cuenta personal, posteriormente podrá crear y compartir webMixs con los estudiantes en los cuales consten los enlaces y recursos a los contenidos preestablecidos. Además de ello, podrá personalizar lecciones de 
aprendizaje, mediante la creación de itinerarios de aprendizaje, el cual puede estar vinculado a diferentes fuentes educativas online, como videos, artículos, sitios web de forma creativa, didáctica e innovadora.

\section{CONCLUSIONES}

De acuerdo con los resultados obtenidos en la investigación el 100\% docentes encuestados manifestaron que requieren más conocimientos y competencias tecnológicas didácticas para impartir el área de matemáticas, ya que, si bien es cierto que utilizan recursos tecnológicos en sus aulas de aprendizaje, desconocen el uso y explotación de herramientas digitales optimas e innovadoras para los procesos matemáticos.

Por otra parte, el $73,53 \%$ de los estudiantes manifestaron que el uso de recursos tecnológicos facilita su proceso de enseñanza-aprendizaje y a su vez instaron a que los docentes implementen en su quehacer educativo diversos recursos que les permita adentrasen aun espacio más real y significativo a fin de consolidar y afianzar sus conocimientos. No obstante, cabe recalcar que la utilización de estos recursos debe denotar como estrategias adicionales en la enseñanza para motivar al estudiantado y darle un rol más protagónico en el proceso, permitiendo que no sea, solo el docente el dueño absoluto de la información, sino que de forma conjunta estudiante-docente puedan lograr la construcción del conocimiento de forma didáctica, creativa y significativa.

Por consiguiente, con esta investigación se buscó fomentar en los docentes la utilización de recursos tecnológicos innovadores como herramientas didácticas para el área de matemática, en la básica superior. En tal sentido se plantearon herramientas digitales como CalcMec, Desmos y Symbaloo, recursos que requieren que los actores estén a la vanguardia en esta nueva sociedad online.

Finalmente es necesario enfatizar que para lograr que los recursos tecnológicos se involucren en el proceso de formación de matemáticas y surtan los efectos ambicionados 
en términos de motivación y aprendizajes significativos, se necesita que el docente diseñe e implemente las herramientas digitales en correlación con los contenidos establecidos en el marco disciplinar del currículo vigente.

\section{FINANCIAMIENTO}

No monetario

\section{AGRADECIMIENTOS}

A la Universidad Católica de Cuenca; por apoyar el desarrollo de la investigación.

\section{REFERENCIAS}

Alvites, C. (2017). Herramientas TIC en el aprendizaje en el área de matemática. [ICT tools in learning in the area of mathematics]. semestral de divulgación científica, $4(1), 18-30$.

Arévalo-Duarte, M., \& Gamboa-Suárez, A. (2015). TIC en el currículo de matemáticas. Una orientación desde el marco de las políticas y proyectos educativos. [ICT in the mathematics curriculum. An orientation from the framework of educational policies and projects]. Rev. Interamericana De Investigación, Educación., 8(1). https://doi.org/10.15332/s1657-107X.2015.0001.07

Barrera-del-Castillo, K. (2015). Entorno virtual para la asignatura enseñanza de las matemáticas en la educación básica. [Virtual environment for the subject teaching mathematics in basic education]. Ra Ximhai, 11(4),315-325.

Coloma, M., Labanda, M., Michay, G., \& Espinosa, W. (2020). Las Tics como herramienta metodológica en matemática. [ICTs as a methodological tool in mathematics]. ESPACIOS, 41(11), 1-7.

Delgado-García, M., García-Prieto, F., \& Gómez Hurtado, I. (2017). Moodle y Facebook como herramientas virtuales didácticas de mediación de aprendizajes: opinión de profesores y alumnos universitarios. [Moodle and Facebook as virtual learning teaching tools of mediation: the opinion of teachers and university students]. Revista Complutense De Educación, 29(3), 807-827. https://doi.org/10.5209/RCED.53968 
María-del-Carmen García-Cárdenas; Darwin Gabriel García-Herrera; Nancy Marcela Cárdenas-Cordero; Juan Carlos Erazo-Álvarez

Domínguez, J, \& Aguilar-Parra, J, \& Fernández-Campoy, J, \& Lozano-Segura, M. (2017). Nuevos recursos tecnológicos para trabajar en un aula de educación infantil: el cuento interactivo considerado un recurso de aprendizaje. [New technological resources to work in a nursery classroom: the interactive story considered a learning resource]. International Journal of Developmental and Educational Psychology, 3(1), 435-448.

Fernández, J., Aguilar, J., Lozano, M. C., \& Domínguez, J. (2017). Nuevos recursos tecnológicos para trabajar en un aula de educación infantil. [New technological resources to work in a nursery classroom]. Internacional de Psicología del Desarrollo y la Educación., 3(1), 435-448. Obtenido de https://n9.cl/ctpfp

González-Ogando, P. (2020). Desmos: una herramienta didáctica para trabajar con funciones y gráficas. [Demos: a didactic tool to work with functions and graphics]. Numeros, didactica de las Matematicas, 104(1), 9-28.

Grisales-Aguirre, A. (2018). Uso de recursos TIC en la enseñanza de las matemáticas: retos y perspectivas. [Use of ICT resources in the teaching of mathematics: challenges and perspectives]. Entramado, 14(2), 198-214. https://doi.org/10.18041/1900-3803/entramado.2.4751

Jiménez-García, J. G., \& Jiménez-Izquierdo, S. (2017). GeoGebra, una propuesta para innovar el proceso enseñanza-aprendizaje en matemáticas. [GeoGebra, a proposal to innovate the teaching-learning process in mathematics]. Revista Electrónica Sobre Tecnología, Educación Y Sociedad, 4(7), 1-17.

López-Neira, L. (2017). Indagación en la relación aprendizaje-tecnologías digitales. [A Look at the Relationship between Learning and Digital Technologies]. Educación y Educadores, 20(1), 91-105. https://dx.doi.org/10.5294/edu.2017.20.1.5

Marcos, E. (2016). Uso de la calculadora gráfica en línea Desmos para la enseñanza de funciones y graficas en $30 \mathrm{ESO}$. [Use of the Desmos online graphing calculator to teach functions and graphs in 30 ESO]. Madrid: UNIR. Recuperado de https://n9.cl/utak

MINEDUC. (2015). Reglamento general a la ley organica de educacion intercultural. [General regulation to the organic law of intercultural education]. Quito: Ministerio de Educacion. Recuperado de https://n9.cl/bca5 
María-del-Carmen García-Cárdenas; Darwin Gabriel García-Herrera; Nancy Marcela Cárdenas-Cordero; Juan Carlos Erazo-Álvarez

Mora, J. (2020). Geogebra como herramienta de transformación educativa en Matemática. [Geogebra as an educative tranformation tool in Maths ]. Mamakuna, $1(14), 70-81$.

Novembre, A, Nicodemo, M, Coll, P. (2015). Matematica y TIC: orientaciones para la enseñanza. [Mathematics and ICT: guidelines for teaching]. Buenos Aires: EDUTEKA. Recuperado de https://n9.cl/r87kp

Soler-Contreras, M, Cárdenas-Salgado, F, Hernández-Pina, F, \& Monroy-Hernández, F. (2017). Enfoques de aprendizaje y enfoques de enseñanza: origen y evolución. [Approaches to Learning and Teaching: Origin and Evolution]. Educación y Educadores, 20(1), 65-88. https://dx.doi.org/10.5294/edu.2017.20.1.4

Torres, P., \& Cobo, J. (2017). Tecnología educativa y su papel en logro de los fines de la educacion. [Educational technology and its role in achieving the aims of education]. Educere, 21(68), 31-40.

Triana-Muñoz, M, Ceballos-Londoño, J, \& Villa-Ochoa, J. (2016). Una dimensión didáctica y conceptual de un instrumento para la Valoración de Objetos Virtuales de Aprendizaje. El caso de las fracciones. [A methodological and conceptual dimension of a tool for the evaluation of Virtual Learning Objects. The case of fractions]. Entramado, 12(2), 186. https://dx.doi.org/10.18041/entramado.2016v12n2.24219 